\title{
Validation of PET Imaging by Alignment to Histology Slices
}

\author{
Philip J. Edwards ${ }^{1}$, Ayman D. Nijmeh ${ }^{2}$, Mark McGurk ${ }^{2}$, Edward Odell ${ }^{3}$, \\ Michael R. Fenlon ${ }^{4}$, Paul K. Marsden ${ }^{5}$, and David J. Hawkes ${ }^{6}$
}

1 Department of Surgical Oncology and Technology, ICL, $10^{\text {th }}$ Floor QEQM Wing, St. Mary's Hospital, London W2 1NY, UK

Eddie.Edwards@imperial.ac.uk

2 Department of Oral and Maxillofacial Surgery, Guy's Hospital, London, UK

3 Division of Oral Medicine and Pathology, KCL, Guy's Hospital, London, UK

${ }^{4}$ Department of Prosthetic Dentistry, Guy's Hospital, London, UK

5 Clinical PET Centre, GKT Medical School, St. Thomas' Hospital, London, UK

${ }^{6}$ Centre for Medical Image Computing, University College, London, UK

\begin{abstract}
The aim of this project is to verify the accuracy of positron emission tomography (PET) in identifying the tumour boundary and eventually to enable PET-guided resection with removal of significantly smaller margins. We present a novel use of an image-guided surgery system to enable alignment of preoperative PET images to postoperative histology. The oral cancer patients must have a high resolution CT scan as well as undergoing PET imaging. Registration of these images to the patient during surgery is achieved using a device that attaches to the patient's upper or lower teeth. During the procedure markers are placed around the lesion within tissue that is to be resected. These are marked along with any convenient anatomical landmarks using the image guidance system, providing the location of the points in the preoperative images. After the sample has been resected, slices through at least 3 of these points are made and photographed. Registration should be possible using these landmarks, but the accuracy of alignment is much improved by marking the bone surface in the histology image and registering to preoperative $\mathrm{CT}$.
\end{abstract}

\section{Introduction}

Histology is generally regarded as the gold standard for identification and labelling of pathological tissues. Imaging techniques can provide a wealth of information about anatomy and physiology in vivo, but its value depends on how closely the image approximates reality. The spatial resolution and level of anatomical or functional detail, however, is much higher in histological slices. Alignment of in vivo images to histology could provide a powerful tool for validation of the imaging process.

Versions of this technique have been used to validate PET. Mega et al registered PET imaging to postmortem brain sections in a patient with Alzheimer's disease [1]. The aim was to compare stained brain sections with a PET scan 
taken 8 hours premortem. A 3D volume was reconstructed from cryosections at $0.5 \mathrm{~mm}$ intervals and aligned to the PET scan using the method of Woods [2]. The stained sections were also registered to the cryosection data using an outlinebased warping algorithm, providing the desired match between stained sections and premortem PET. Humm et al, on the other hand, inserted small rods into a tumour to act as markers for registration of magnetic resonance imaging (MRI) or PET images to histology in rodents 3. Malandain et al produced a system for alignment of MRI to autoradiographs in tumour bearing mice [4. Here an initial 3D volume of the autoradiographs is constructed and registered to the MRI image. The alignment for each autoradiographic slice is then refined and a new volume produced, and this process is repeated until a sufficiently good match is achieved.

In all these examples a volume is produced by aligning many histological slices from a postmortem subject. The aim of this project is to examine the accuracy of PET imaging for delineation of tumours in patients, for which we require a means of alignment to the excised tissue. This sample may not be able to provide many parallel slices, so a method that can register a single slice is required.

\section{Method}

The patient population considered for evaluation had an oral cancer sited in close association with the bone of the upper or lower jaw. A lockable acrylic dental stent (LADS) was made to clamp firmly to the patient's upper or lower teeth.

Fiducial markers can be attached to the LADS which provide accuracy of registration for the head, both in terms of stability and repeatability of positioning, to a tolerance of $1-4 \mathrm{~mm}$ [5. Any rotational instability causes greater errors at a distance from the teeth. We would expect accuracy in these cases to be higher, since the lesion is always located very close to the LADS.

\subsection{Preoperative Image Acquisition and Processing}

Preoperatively, prior to undergoing a PET scan, the LADS and fiducial frame are attached to the patient and the imaging markers filled with $5 \mathrm{kBq} / \mathrm{ml}$ FDG. This is followed by a CT scan, with the markers filled with iodine solution. Usually there are 8 markers visible in both scans which are used for registration (see Fig. 1).

The position of the fiducials is marked in both sets of images. This provides registration from CT to PET. This was found to be a more accurate and reliable method than automated voxel-based registration [6], since the amount of activity in the PET scan can be quite small unless a significant amount of brain is included and there may be relative movement between the head and the region of interest if the lesion is in the mandible.

The PET scan is segmented using Analyze [7] to remove any activity other than the lesion. A set of surfaces is then produced using marching cubes [8] for 

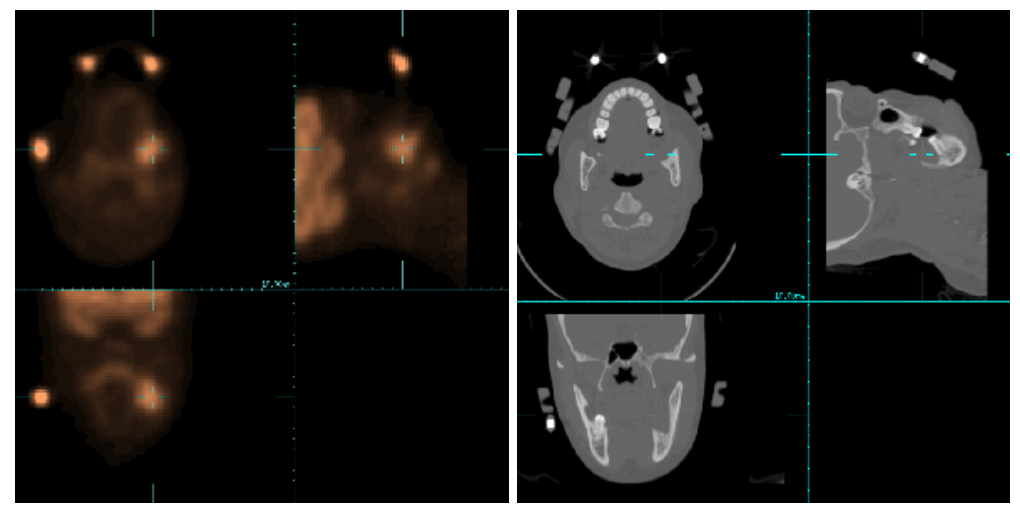

Fig. 1. An example PET scan and CT scan, showing the visibility of the fiducial markers

different thresholds to produce PET models of the lesion. These will be used to produce PET isointensity contours on the resulting histology slice. A bone and teeth surface model is created from the CT scan, also by marching cubes. This is useful for checking registration accuracy and is also required for the outline-based registration step described later.

\subsection{Registration to the Physical Space of the Patient}

The LADS is designed to carry a tracking frame of 6 infra-red emitting diodes (IREDs). that are located by the Optotrak position sensor from Northern Digital, Inc. Both the tracker and the fiducial frame are attached and the imaging fiducials are replaced by physical markers that dock accurately with a tracked pointer. Marking these points provides their location in the physical coordinate system defined by the IRED tracking frame. Registration to the same points marked in the PET and CT images aligns the preoperative data to the physical space defined by this frame. Now the wings carrying the fiducials can be removed and the intraoperative alignment is performed simply by attaching the LADS to the patient's teeth.

The beauty of using the LADS for this purpose is that the image-to-physical registration process can take place without the patient being present, as long as both the tracking frame and fiducial frame are attached.

It proved instructive to attach the cast of the teeth to the LADS. Then a pointer-based guidance system can be used on the cast to check that the CT is well aligned by marking bone surface points. The lesion can be visualised to check PET alignment (Fig. 2(c)). These planning checks save on operating room time and forewarn of problems with the data.

At surgery only the LADS with the tracking frame is attached to the teeth. The LADS locates and locks onto the teeth with great accuracy [5]. The position of the lesion can now be visualised using pointer-based guidance or augmented reality visualisation (see Fig. 2(d)). 


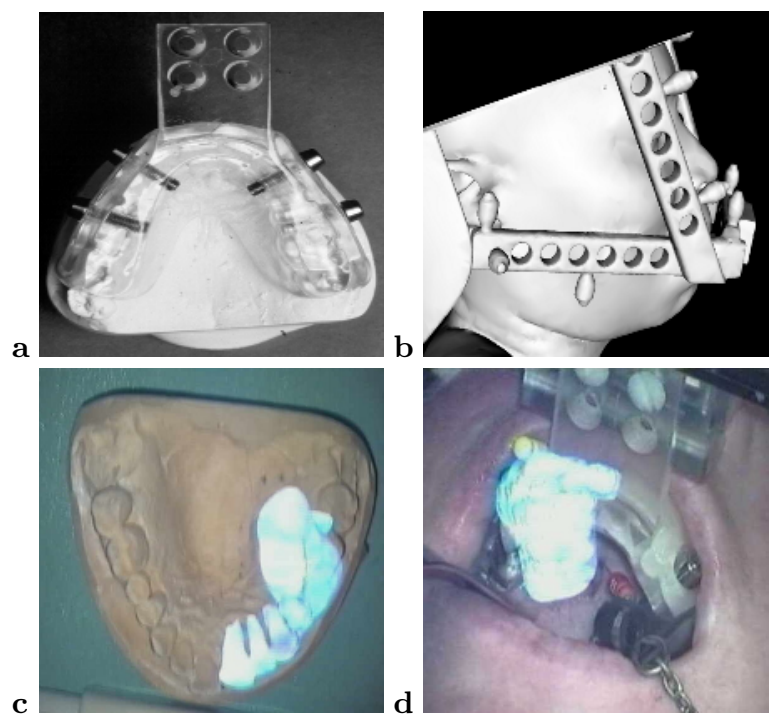

Fig. 2. The LADS registration device, showing attachment to the teeth (a), a rendering of the fiducial frame from CT (b), an overlay on the cast taken when producing the LADS (c) and an overlay during the operation showing that marker pins have been placed around the lesion $(\mathrm{d})$.

\subsection{Intraoperative Marker Placement}

The surgeon and pathologist decide from the preoperative renderings the position of likely cuts through the lesion for histological assessment of the lesion. We then aim to place markers so that they define this plane, though high accuracy at this stage is not important. At least 3 points are required to define the plane of the cut. If anatomical landmarks are available, such as the cusp of a given tooth, these can be used. Otherwise markers are implanted in the tissue around the lesion. Dental barbed broaches or preferably standard bone screws are used for this purpose.

The screws are then marked with the image-guided surgery system, providing their position in the preoperative images. We use the CT scan as the reference image. The position of all intraoperative markers are recorded.

The surgery then proceeds and the sample containing the lesion and all the markers is removed in one piece to be transferred to the pathology laboratory. It is normal practice for the tumour and a $2 \mathrm{~cm}$ margin of normal surrounding tissue to be removed.

\subsection{Postopertative Histology}

The tumour specimen is formalin fixed. With the aid of renderings showing the bone from CT, lesion from PET and the location of the markers, a number of cuts are made through the specimen as close as possible to at least 3 landmarks. 
The cuts are made with an Exact $0.3 \mathrm{~mm}$ thickness diamond grinding band. This part of the process relies on operator skill, but with experience it is possible to produce a cut through the relevant points that is very close to planar. The diamond band should not produce significant distortion of the tissue, especially since we have chosen non-mobile lesions close to the bone.

After each cut the sample is photographed with a Nikon coolpix 900 digital camera set on macro. The plane of the cut is manually placed to be perpendicular to the optical axis of the camera, which is mounted on a camera stand. Two rulers are placed in the same plane as the sample to be used for scaling of the resulting image. The position of the landmarks and the outline of the tumour are identified by the pathologist in the histology image. The landmark positions are released for registration purposes, but the tumour outline is not disclosed until the registration and PET rendering is completed.

\subsection{Registration and Refinement}

Registration can now be simply performed by point-based alignment of the landmarks from the scaled histology image to the landmark positions in the preoperative images. This process was prone to errors of rotation. The guidance system should be accurate to around $1 \mathrm{~mm}$, but the fiducial points are often only a few $\mathrm{mm}$ apart. Every effort is made to ensure landmarks are well spread in a triangle, but often this is not possible as the available anatomy at the beginning of surgery may consist of only one tooth. The close proximity of the landmarks can produce significant rotational errors.

To refine the registration the outline of the bone is delineated manually in the histology slice and registered to the bone surface from CT using the iterative closest point (ICP) method [9]. The result of the point-based registration is used as a starting estimate.

\subsection{Validation}

The technique described here could be used as a validation of PET imaging for tumour localisation, but the method of alignment itself should be validated. Unfortunately there is no easy gold standard registration of PET to histology with which to assess the current method.

It is possible to examine the precision of the registration, however. This is performed for the landmark identification by marking the fiducials intraoperatively six times. For the ICP registration refinement we use multiple random starting estimates within the range of error associated with the landmark registration.

\section{Results}

An example resulting alignment is shown in figure 3. The outline on the histology slice was provided by the pathologist. Bone from CT and the tumour from PET are overlaid on the histology image using landmark registration (Fig. 3(b)) and after ICP registration (Fig. 3(c)). For landmark registration there is a clear 


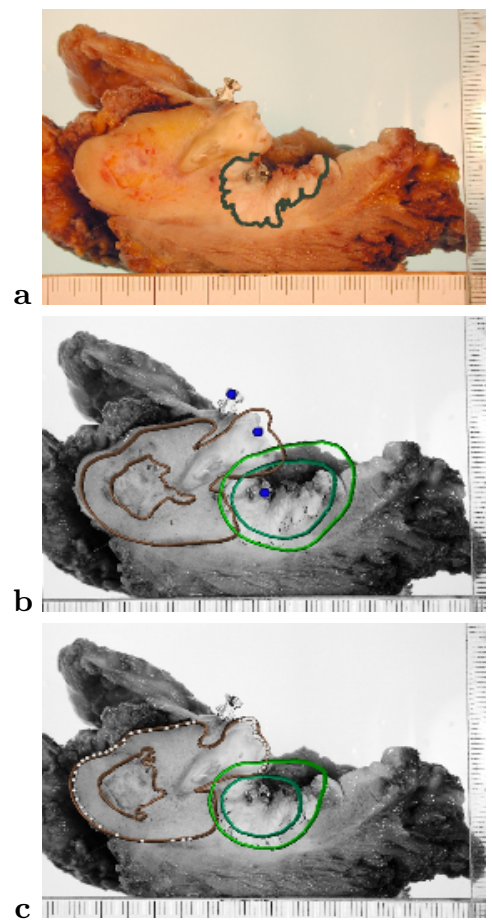

Fig. 3. An example histology slice showing the outline of the lesion (a), an overlay of the bone (brown) and lesion (green) after registration by landmarks (b) and ICP (c). The latter clearly gives a visually better alignment. Two thresholds are used that roughly correspond to the inner and outer boundary of the lesion in PET, demonstrating how we can use this method to help determine how best to use PET for tumour segmentation.

misalignment of the bone surface. After ICP the alignment is visually much improved. Figure 4 shows the position of the histology slice using the two registrations displayed over a rendering of the bone surface from $\mathrm{CT}$ and the lesion from PET. The rotational discrepancy between the two registrations is clear.

Repeated marking of the fiducials with the image-guided surgery system gave errors of around $1 \mathrm{~mm}$. The RMS residual error on the bone surface points after ICP is $0.34 \mathrm{~mm}$. Repeated ICP registrations based on random starting positions were highly consistent, with a mean target registration error (TRE) within the histology slice of $0.54 \mathrm{~mm}$. It should be stressed that this is an initial result on one dataset and should not be taken as a true measure of the accuracy of the method.

\section{Discussion}

We have presented a method for alignment of PET imaging to histology slices. This should enable validation of the ability to delineate a tumour using PET. 
An initial result is given in which the alignment precision after ICP registration appears to be around $0.5 \mathrm{~mm}$. This is an early result and further experimentation is required. The effect of outline accuracy on precision could be estimated by marking multiple boundaries. Also a true gold standard may be possible with phantom or animal studies. Particularly a series of patients needs to be examined using the system. The initial results do suggest that the use of a guidance system in conjunction with a CT scan to provide the bone surface enables accurate alignment between PET and histology.

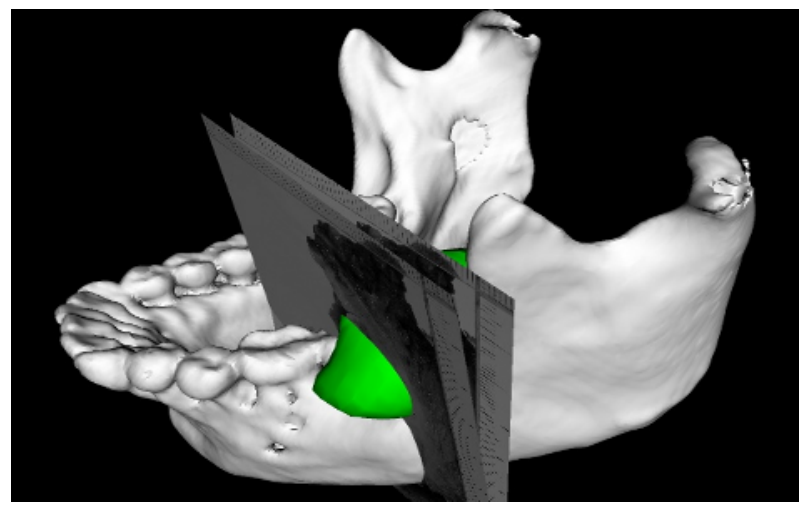

Fig. 4. A rendering of the mandible from CT, lesion from PET and the histology slice, registered using landmarks and ICP. The rotational error between the two registrations can clearly be seen. We believe the ICP result provides accurate alignment.

There are a few caveats when considering this process. Firstly, if the PET scan is taken a significant time before surgery there is the possibility that the lesion may have grown. To minimise this effect scanning was performed 1 week before surgery so as not to allow significant time for tumour growth. In general scanning should take place as near as possible to the time of the operation. Another potential problem is the shrinkage of soft tissue on removal or fixation. By choosing non-mobile lesions that are close to bone, we not only have the advantage of being able to match to the bone surface from CT, but also any deformation of the soft tissue is likely to be minimised. Shrinkage on fixation across the tumour was experimentally assessed to be of the order of $1 \%$ linear based on measurement along needles passed through the lesion.

The aim of the project will be to collect a database of PET images aligned to histology slices. This will enable quantitative analysis of the accuracy of PET segmentation techniques for tumour boundary delineation. Once we have confidence in the accuracy of the tumour segmentation we can develop a PET-based image-guided surgery system that will allow complete resection of the lesion with much smaller margins. The current practice is to ensure full resection by taking a significant margin of $1-3 \mathrm{~cm}$. Accurate PET-guided tumour resection should result in less-invasive surgery and reduced morbidity for the patient. 
This paper presents, to the best knowledge of the authors, the first attempt to use image-guided surgery techniques to perform alignment of PET imaging to histology for verification of tumour boundary segmentation.

\section{References}

1. Mega, M.S., Chen, S.S., Thompson, P.M., Woods, R.P., Karaca, T.J., Tiwari, A., Vinters, H.V., Small, G.W., Toga, A.W.: Mapping histology to metabolism: Coregistration of stained whole-brain sections to premortem pet in alzheimer's disease. Neuroimage 5 (1997) 147-153

2. Woods, R.P., Mazziotta, J.C., Cherry, S.R.: MRI-PET registration with automated algorithm. J. Comput. Assist. Tomogr. 17 (1993) 536-546

3. Humm, J.L., Ballon, D., Hu, Y.C., Ruan, S., Chui, C., Tulipano, P.K., Erdi, A., Koutcher, J., Zakian, K., Urano, M., Zanzonico, P., Mattis, C., Dyke, J., Chen, Y., Harrington, P., O'Donoghue, J.A., Ling, C.C.: A stereotactic method for the threedimensional registration of multi-modality biologic images in animals: NMR, PET, histology, and autoradiography. Med. Phys. 30 (2003) 2303-2314

4. Malandain, G., Bardinet, E., Nelissen, K., Vanduffel, W.: Fusion of autoradiographs with an MR volume using 2-D and 3-D linear transformations. Neuroimage 23 (2004) 111-127

5. Fenlon, M.R., Jusczyzck, A.S., Edwards, P.J., King, A.P.: Locking acrylic resin dental stent for image-guided surgery. J. Prosthet. Dent. 83 (2000) 482-485

6. Studholme, C., Hill, D.L.G., Hawkes, D.J.: Automated 3D registration of MR and CT images of the head. Med. Image Anal. 1 (1996) 163-175

7. Robb, R.A., Hanson, D.P., Karwoski, R.A., Larson, A.G., Workman, E.L., Stacy, M.C.: ANALYZE: a comprehensive, operator-interactive software package for multidimensional medical image display and analysis. Comput. Med. Imaging Graphics 13 (1989) 433-454

8. Lorensen, W.E., Cline, H.E.: Marching cubes: A high resolution 3D surface reconstruction algorithm. Comput. Graph. 21 (1987) 163-169

9. Besl, P.J., McKay, N.D.: A method for registration of 3-D shapes. IEEE Trans. Pattern Anal. Mach. Intell. 14 (1992) 239-256 